\title{
Oral hygiene, prevalence of gingivitis, and associated risk factors among pregnant women in Sarlahi District, Nepal
}

\author{
D. J. Erchick ${ }^{1 *}$, B. Rai ${ }^{2}$, N. K. Agrawal ${ }^{3}$, S. K. Khatry², J. Katz ${ }^{1}$, S. C. LeClerq ${ }^{1,2}$, M. A. Reynolds ${ }^{4}$ and L. C. Mullany ${ }^{1}$
}

\begin{abstract}
Background: The oral health status of pregnant women in low-resource communities such as Nepal has not been well characterized. This sub-population is also of specific interest given associations between poor oral health and adverse pregnancy outcomes previously documented in other settings. We explored relationships between gingivitis and risk factors among pregnant women in rural Nepal.

Methods: The design was a community-based, cross-sectional study in a sub-area of Sarlahi District, Nepal. Pregnant women $<26$ weeks gestation underwent clinical periodontal exams conducted by community-based oral health workers. Exams included a full mouth assessment measuring bleeding on probing (BOP), probing depth (PD) (six sites per tooth), and gingival recession, the distance from the cemento-enamel junction to the free gingival margin (two direct sites per tooth). Data on participant risk factors were collected through household surveys, including demographic characteristics, oral health behaviors, care seeking, and health attitudes. Multivariable logistic regression modeling was used to assess relationships between gingivitis and risk factors.

Results: We enrolled 1452 participants, of which 40\% $(n=582)$ had signs of clinical gingivitis and 60\% ( $n=870)$ clinical health. Average participant age was 23. Most participants (88\%) had never received oral health care. Participants averaged $10 \%$ of sites with BOP with most (79\%) having $\geq 1$ site with BOP. Nine percent of participants had $\geq 1$ site with PD $\geq 4 \mathrm{~mm}$, although very few participants $(0.7 \%)$ had sites with PD $\geq 5 \mathrm{~mm}$. Few participants (13\%) had any recession $(\geq 1 \mathrm{~mm})$. In the final adjusted model, odds of gingivitis increased by $3 \%$ for each year of age (aOR $1.03,95 \% \mathrm{Cl} 1.00,1.06)$ and were higher for women of short maternal stature $(<150 \mathrm{~cm})$ (aOR $1.43,95 \% \mathrm{Cl}: 1.14,1.79)$ and among women reporting cost to be a barrier to seeking dental care (aOR 2.13 , 95\% Cl: 1.09, 4.15).
\end{abstract}

Conclusions: Gingivitis was common and associated with age, maternal stature, self-reported high cost of dental care, and other risk factors among pregnant women in rural Nepal.

Trial registration: ClinicalTrials.gov Identifier: NCT01177111 (Nepal Oil Massage Study) and NCT02788786 (Pilot Trial).

Keywords: Nepal, Gingivitis, Oral health, Oral health behaviors, Dental care seeking behavior, Pregnancy

\footnotetext{
*Correspondence: derchick@gmail.com

${ }^{1}$ Department of International Health, Johns Hopkins Bloomberg School of

Public Health, Baltimore, MD, USA

Full list of author information is available at the end of the article
}

(c) The Author(s). 2019 Open Access This article is distributed under the terms of the Creative Commons Attribution 4.0 International License (http://creativecommons.org/licenses/by/4.0/), which permits unrestricted use, distribution, and reproduction in any medium, provided you give appropriate credit to the original author(s) and the source, provide a link to the Creative Commons license, and indicate if changes were made. The Creative Commons Public Domain Dedication waiver (http://creativecommons.org/publicdomain/zero/1.0/) applies to the data made available in this article, unless otherwise stated. 


\section{Background}

Periodontal disease includes a group of inflammatory conditions, typically initiated by oral bacteria, progressing from reversible accumulation of plaque and inflammation of gingival tissue (gingivitis) to irreversible breakdown of the supportive tissues of the teeth and eventually tooth loss (periodontitis) [1]. Globally, gingivitis is common and severe periodontitis affects 10 to $15 \%$ of adult populations [2]. Risk factors for periodontal disease include older age, poor oral hygiene, tobacco use, alcohol consumption, stress, malnutrition, and diabetes mellitus and other chronic diseases, although these have been evaluated primarily in populations in high-income countries [3]. Associations have been observed between periodontal disease and distal factors such as income and education, urban/rural location, race, and ethnicity [4]. Periodontal disease has been implicated as a potential cause of systemic conditions, such as adverse pregnancy outcomes [5].

Although periodontal health is recognized as an important component of general well-being, oral health remains neglected in many low- and middle-income countries and among high-risk and underserved sub-populations in some high-income countries $[6,7]$. In many low-income countries, oral health facilities, equipment, and personnel are in short supply, severely limiting access to care, especially preventative services [7]. Nepal, for example, has only 2 dentists per 100,000 population, one of the lowest ratios among South Asian countries [8]. Few studies have assessed the oral health and oral hygiene practices of adult Nepalis. A review of four surveys conducted in the 1980s concluded that Nepal ranked among the bottom 15\% of countries in terms of periodontal conditions [9]. A more recent national survey (2004) showed some improvement; Community Periodontal Index of Treatment Needs (CPITN) scores among 15-16 year-olds were $26 \%$ periodontal health (score 0 ), $8 \%$ bleeding on probing (BOP) (score 1), 61\% BOP and calculus (score 2), 5\% probing depth (PD) 4-5 mm (score 3), and 0\% PD $\geq 6 \mathrm{~mm}$ (score 4). Among adults 35-44 years, the corresponding scores were $7 \%$ periodontal health (score 0 ), $3 \%$ BOP (score 1), 27\% BOP and calculus (score 2), 48\% PD 4-5 mm (score 3 ), and $16 \% \mathrm{PD} \geq 6 \mathrm{~mm}$ (score 4) [10-12].

The oral health status of pregnant women in low-resource communities such as Nepal has not been well characterized. This sub-population is also of specific interest given previously documented associations between poor oral health and adverse pregnancy outcomes $[5,13]$. In this study, we explored relationships between demographic characteristics, oral hygiene practices, care-seeking, knowledge, and gingivitis among pregnant women in the Terai region of Nepal.

\section{Methods}

Participants in this study were enrolled in the Nepal Oral Health Cohort Study (NOHCS), a community-based, prospective cohort study of gingivitis and adverse pregnancy outcomes. NOHCS participants were identified and determined eligible using the infrastructure of a large community-based randomized trial, the Nepal Oil Massage Study (NOMS) (NCT01177111), which was actively enrolling a population-based sample of pregnant women in this study area.

\section{Oral health examination}

Oral health examinations were performed by five auxiliary nurse-midwives, with no previous experience in dentistry or clinical research, who were trained as "community-based oral health workers" for this study by an experienced dentist (NKA) at the Department of Dentistry, Institute of Medicine, Tribhuvan University, Kathmandu, Nepal. Training for the oral health workers included identification of plaque and calculus, signs of gingivitis, and measurement of PD, $\mathrm{BOP}$, and gingival recession, the distance from the cemento-enamel junction (CEJ) to the free gingival margin (CEJ-GM). Oral health field workers were also trained in clinical research methods and ethics for human subjects research. Training lasted 3-4 weeks and included classroom instruction and practice of periodontal techniques under the guidance of the dentist. We estimated the validity of PD measurements of the oral health workers relative to the dentist and found that percent agreement, weighted kappa scores, and intraclass correlation coefficients, with an allowance of PD $\pm 1 \mathrm{~mm}$, exceeded 99\%, 0.7, and 0.9 , respectively, indicating an acceptable level of agreement.

Periodontal measurements were made using a color Williams probe (Hu-Friedy, Chicago, IL, USA). PD was measured on six sites per tooth (disto-, mid-, and mesial- aspects of buccal and lingual surfaces) and the CEJ-GM distance on two sites per tooth (mid- buccal and lingual aspects), excluding third molars. After probing each quadrant, the presence or absence of BOP was recorded for buccal and lingual surfaces of each tooth. PD values were recorded in millimeters from 1 to 10 , rounded to the next higher whole number. CEJ-GM distances were recorded similarly, with values of 0 to 10 $\mathrm{mm}$. If the free gingiva was coronal to the CEJ, the CEJ-GM measurement was recorded as 0. Clinical attachment loss (CAL) was calculated by summing the PD and CEJ-GM distance; the CEJ-GM distance was assigned a value of 0 for distal and mesial sites, where this measure was not collected, and these sites were not considered in measures of CAL. All scores were 
recorded on paper forms by a trained assistant, and electronically entered by experienced data entry operators.

Clinical periodontal disease was defined according to a classification scheme developed by the American Academy of Periodontology and European Federation of Periodontology to update the 1999 classification of periodontal diseases and conditions [14]. A case of clinical health was defined as a participant with all sites $\mathrm{PD} \leq 3$ $\mathrm{mm}$ and $\mathrm{BOP}<10 \%[15,16]$. A case of clinical gingivitis was defined as a participant with $\mathrm{BOP} \geq 10 \%$, stratified as localized gingivitis (BOP 10-30\%) and generalized gingivitis (BOP $\geq 30 \%$ ) $[15,16]$. Among localized and generalized gingivitis categories, we further stratified participants by those with $\geq 1$ site with $P D \geq 4 \mathrm{~mm}$. Our descriptive and inferential analyses compared participants with clinical health vs. clinical gingivitis. Other periodontal characteristics, including prevalence of potential clinical signs of mild periodontitis, were also described [17-20].

\section{Oral hygiene questionnaire}

Data on participant demographics, oral hygiene practice, care-seeking, knowledge, and other characteristics were collected in the home, through a series of questionnaires administered over the course of pregnancy. Questionnaires were developed jointly by the authors and senior research staff, who are experienced in the conduct of community-based public health research and resident in the study population. Pre-tests of the questionnaires were conducted among study research staff, although the instrument's validity and reliability were not formally evaluated. Questionnaires were administered in Maithili or Nepali language, depending on the participant's primary language, in the home of participants by female study workers who were locally resident in the study area.

\section{Statistical analysis}

Descriptive bivariate analyses between participant characteristics and the outcome, gingivitis, were evaluated using t-tests and logistic regression for continuous and binary/categorical variables, respectively. We ran a series of logistic regression multivariable models, sequentially including risk factor variables from four broad domains: maternal characteristics, oral hygiene behaviors, oral health knowledge and attitudes, and socioeconomic characteristics. Covariates were selected for inclusion in these regression models by examining bivariate relationships with gingivitis status using a cutoff of $p<0.10$. Adjusted odds ratios (aOR) and 95\% confidence intervals (CI) were calculated. All statistical analyses were performed in STATA 14.2 (StataCorp, College Station, TX, USA).

\section{Ethical review}

This study received ethical approval from the Institutional Review Board at Johns Hopkins Bloomberg School of Public Health (Baltimore, USA) and the Ethical Review Board of the Nepal Health Research Council (Kathmandu, Nepal).

\section{Results}

Between January 11, 2016, and November 26, 2016, among 1458 eligible pregnant women approached, 6 refused participation, leaving a total of 1452 participants. Of these, 32 were lost to follow-up (30 could not be contacted and 2 could not be interviewed because they had moved outside the study area) after the periodontal examination. Therefore, these participants provided data on their periodontal health but not on oral health risk factors for this analysis. Average participant age was 23.1 (SD: 4.7) ranging from 15 to 41, and the mean gestational age of pregnant women at enrollment was 14 weeks, ranging from 6 to 25 weeks (Table 1). About two-thirds $(n=934,64 \%)$ of women had a normal BMI, $29 \%(n=428)$ were underweight, and only $6 \%(n=90)$ overweight or obese. Roughly three-quarters of participants $(n=1049,72 \%)$ had at least one previous pregnancy, while $28 \%(n=403)$ of women had not been pregnant before. Literacy among the study population was low $(n=671,46 \%)$ and more than half of women had never attended school $(n=783,54 \%)$. A majority of women lived in homes constructed of thatch, sticks, or bamboo $(n=897,62 \%)$, versus wood planks, brick, or stone $(n=555,38 \%)$. Women were likely to have some electricity available in the home $(n=1338,92 \%)$. Nearly all women had access to a well for drinking water $(n=$ $1446,>99 \%)$. Almost half of women had no access to a latrine $(n=624,43 \%)$.

\section{Periodontal disease status}

The majority of women $(n=1224,84 \%)$ had all 28 teeth (ignoring third molars) (Table 2). Most participants $(n=$ $1151,79 \%)$ had at least one site with BOP, and participants averaged $10 \%$ of sites with BOP, the equivalent of 17 tooth sites (median $=9$ ) per participant. Mean PD was $1.7 \mathrm{~mm}$ (SD: 0.3 ) and max PD ranged from $2 \mathrm{~mm}$ to $7 \mathrm{~mm}$. Nine percent $(n=125)$ of participants had at least 1 site with $\mathrm{PD} \geq 4 \mathrm{~mm}$, although very few participants ( $n=10,0.7 \%$ ) had sites with PD $\geq 5 \mathrm{~mm}$. Mean CAL was equivalent to mean $\mathrm{PD}$, and nearly all of $\mathrm{CAL} \geq 4 \mathrm{~mm}$ was due to periodontal pocketing secondary to gingival enlargement, indicating very little recession of the gingiva in this population. Only $13 \%(n=184)$ of participants had at least one site with recession. 
Table 1 Demographic characteristics of participants in the oral hygiene study

\begin{tabular}{|c|c|c|c|c|}
\hline Characteristic & All & Clinical health & Clinical gingivitis & Mean difference / OR $(95 \% \mathrm{Cl})^{\mathrm{a}}$ \\
\hline \multicolumn{5}{|l|}{ Maternal age } \\
\hline Year (mean, \pm SD) & $23.1 \pm 4.7$ & $22.8 \pm 4.6$ & $23.6 \pm 4.8$ & $0.8(0.3,1.3)$ \\
\hline \multicolumn{5}{|l|}{ Maternal age (years) } \\
\hline$<18$ & $157(10.8)$ & $115(13.2)$ & $42(7.2)$ & $0.51(0.35,0.74)$ \\
\hline $18-<35$ & $1267(87.3)$ & 739 (84.9) & $528(90.7)$ & Ref \\
\hline$\geq 35$ & $28(1.9)$ & $16(1.8)$ & $12(2.1)$ & $1.05(0.49,2.24)$ \\
\hline \multicolumn{5}{|l|}{ Maternal height } \\
\hline Cm (mean, $\pm \mathrm{SD})$ & $150.8 \pm 5.5$ & $151.2 \pm 5.4$ & $150.2 \pm 5.6$ & $-1.0(-1.6,-0.4)$ \\
\hline \multicolumn{5}{|l|}{ Maternal weight } \\
\hline $\mathrm{Kg}($ mean, $\pm \mathrm{SD})$ & $45.8 \pm 7.1$ & $46.1 \pm 7.2$ & $45.4 \pm 6.8$ & $-0.7(-1.5,0.0)$ \\
\hline \multicolumn{5}{|l|}{ Maternal BMI } \\
\hline Underweight & $428(29.5)$ & $256(29.4)$ & $172(29.6)$ & $1.01(0.80,1.27)$ \\
\hline Normal weight & $934(64.3)$ & $560(64.4)$ & $374(64.3)$ & Ref \\
\hline Overweight or obese & $90(6.2)$ & $54(6.2)$ & $36(6.2)$ & $1.00(0.64,1.55)$ \\
\hline \multicolumn{5}{|l|}{ Ethnic group } \\
\hline Hills (Pahadi) & $106(7.3)$ & $54(6.2)$ & $52(8.9)$ & Ref \\
\hline Plains (Madeshi) & $1346(92.7)$ & $816(93.8)$ & $530(91.1)$ & $0.67(0.45,1.00)$ \\
\hline \multicolumn{5}{|l|}{ Gravidity } \\
\hline First pregnancy & $403(27.8)$ & $260(29.9)$ & $143(24.6)$ & $0.78(0.61,0.99)$ \\
\hline 1-3 previous pregnancies & $872(60.1)$ & $511(58.7)$ & $361(62.0)$ & Ref \\
\hline$\geq 4$ previous pregnancies & $177(12.2)$ & 99 (11.4) & 78 (13.4) & $1.12(0.81,1.54)$ \\
\hline \multicolumn{5}{|l|}{ Maternal literacy } \\
\hline No & 781 (53.8) & $455(52.3)$ & $326(56.0)$ & Ref \\
\hline Yes & $671(46.2)$ & $415(47.7)$ & $256(44.0)$ & $0.86(0.70,1.06)$ \\
\hline \multicolumn{5}{|l|}{ Maternal education (years) } \\
\hline 0 & $783(54.0)$ & $456(52.5)$ & $327(56.2)$ & Ref \\
\hline $1-9$ & $404(27.8)$ & $248(28.5)$ & $156(26.8)$ & $0.88(0.69,1.12)$ \\
\hline$\geq 10$ & $264(18.2)$ & $165(19.0)$ & $99(17.0)$ & $0.84(0.63,1.11)$ \\
\hline \multicolumn{5}{|l|}{ Electricity } \\
\hline No & $114(7.9)$ & $62(7.1)$ & $52(8.9)$ & Ref \\
\hline Yes & $1338(92.1)$ & $808(92.9)$ & $530(91.1)$ & $0.78(0.53,1.15)$ \\
\hline \multicolumn{5}{|l|}{ House construction material } \\
\hline None, thatch, sticks, or bamboo & $897(61.8)$ & $526(60.5)$ & $371(63.7)$ & Ref \\
\hline Wood planks, bricks, or stone & $555(38.2)$ & $344(39.5)$ & $211(36.3)$ & $0.87(0.70,1.08)$ \\
\hline \multicolumn{5}{|l|}{ House roof material } \\
\hline None, plastic, thatch, or grass & $111(7.6)$ & $58(6.7)$ & $53(9.1)$ & Ref \\
\hline Tile, tin, or concrete & $1341(92.4)$ & $812(93.3)$ & $529(90.9)$ & $0.71(0.48,1.05)$ \\
\hline \multicolumn{5}{|l|}{ Latrine } \\
\hline No latrine & $624(43.0)$ & $356(40.9)$ & $268(46.0)$ & Ref \\
\hline Brick, concrete, or pit latrine & $828(57.0)$ & $514(59.1)$ & $314(54.0)$ & $0.81(0.66,1.00)$ \\
\hline
\end{tabular}

Data presented as No. (\%) unless otherwise noted

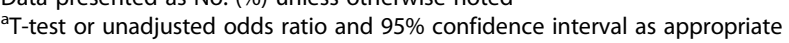

Statistically significant for a mean difference are in bold italic

Statistically significant for an odds ratio are in bold 
Table 2 Clinical periodontal characteristics of participants in the oral hygiene study

\begin{tabular}{|c|c|}
\hline Characteristic & No., $\%$ \\
\hline \multicolumn{2}{|l|}{ Number of teeth ${ }^{a}$} \\
\hline Mean number of teeth (mean \pm SD) & $27.7 \pm 0.8$ \\
\hline \multicolumn{2}{|l|}{ Clinical health and gingivitis } \\
\hline Health (All sites PD $\leq 3 \mathrm{~mm}$ and $\mathrm{BOP}<10 \%$ ) & $870(59.9)$ \\
\hline Gingivitis (BOP $\geq 10 \%$ and/or PD $\geq 4 \mathrm{~mm}$ ) & $582(40.1)$ \\
\hline - Localized gingivitis (BOP < 30\%) & $468(80.4)$ \\
\hline - Proportion with no sites PD $\geq 4 \mathrm{~mm}$ & $373(79.7)$ \\
\hline - Proportion with $\geq 1$ sites PD $\geq 4 \mathrm{~mm}$ & $95(20.3)$ \\
\hline - Generalized gingivitis (BOP $\geq 30 \%$ ) & $114(19.6)$ \\
\hline - Proportion with no sites PD $\geq 4 \mathrm{~mm}$ & $84(73.7)$ \\
\hline - Proportion with $\geq 1$ sites PD $\geq 4 \mathrm{~mm}$ & $30(26.3)$ \\
\hline \multicolumn{2}{|l|}{ Bleeding on probing (BOP) } \\
\hline Percent of sites BOP (mean \pm SD) & $10.2 \pm 12.2$ \\
\hline No sites BOP & $301(20.7)$ \\
\hline$\geq 1$ site $\mathrm{BOP}$ & $1151(79.3)$ \\
\hline$\geq 1$ site $\mathrm{BOP} \& \mathrm{BOP}<10 \%$ & $621(42.8)$ \\
\hline $\mathrm{BOP} \geq 10 \% \& \mathrm{BOP}<30 \%$ & $416(28.7)$ \\
\hline $\mathrm{BOP} \geq 30 \%$ & $114(7.9)$ \\
\hline \multicolumn{2}{|l|}{ Probing depth (PD) } \\
\hline Mean PD (mm) (mean \pm SD) & $1.7 \pm 0.3$ \\
\hline Mean PD at direct sites $(\mathrm{mm})($ mean $\pm \mathrm{SD})$ & $1.5 \pm 0.2$ \\
\hline Percent of sites PD $\geq 4 \mathrm{~mm}$ (mean \pm SD) & $0.2 \pm 1.0$ \\
\hline$\geq 1$ site $P D \geq 4 \mathrm{~mm}$ & $125(8.6)$ \\
\hline$\geq 3$ sites $P D \geq 4 \mathrm{~mm}$ & $40(2.8)$ \\
\hline$\geq 1$ site $P D \geq 5 \mathrm{~mm}$ & $10(0.7)$ \\
\hline$\geq 3$ sites $P D \geq 5 \mathrm{~mm}$ & $3(0.2)$ \\
\hline \multicolumn{2}{|l|}{ Clinical attachment loss (CAL) ${ }^{b}$} \\
\hline Mean CAL at direct sites $(\mathrm{mm})($ mean $\pm \mathrm{SD})$ & $1.7 \pm 0.3$ \\
\hline$\geq 1$ site recession $\geq 1 \mathrm{~mm}$ & $184(12.7)$ \\
\hline$\geq 1$ site $C A L \geq 4 \mathrm{~mm}$ & $206(14.2)$ \\
\hline$\geq 3$ site $C A L \geq 4 \mathrm{~mm}$ & $72(5.0)$ \\
\hline$\geq 1$ site $C A L \geq 5 \mathrm{~mm}$ & $65(4.5)$ \\
\hline$\geq 3$ site $C A L \geq 5 \mathrm{~mm}$ & $14(1.0)$ \\
\hline$\geq 1$ site $C A L \geq 6 \mathrm{~mm}$ & $21(1.5)$ \\
\hline$\geq 3$ site $C A L \geq 6 \mathrm{~mm}$ & $3(0.2)$ \\
\hline Percent of CAL $\geq 4 \mathrm{~mm}$ due to pocketing (mean \pm SD) & $99.6 \pm 1.5$ \\
\hline Percent of $C A L \geq 4 m m$ due to recession (mean \pm SD) & $0.3 \pm 1.5$ \\
\hline
\end{tabular}

Sixty percent of participants $(n=870)$ fit the definition of clinical health and $40 \%(n=582)$ clinical gingivitis. Among women with gingivitis, $80 \%(n=468 / 582)$ had localized gingivitis and 20\% $(n=114 / 582)$ generalized gingivitis. The proportion of sites with $\mathrm{PD} \geq 4 \mathrm{~mm}$ in women with localized and generalized gingivitis were similar $(n=95 / 468,20 \%$ and $n=30 / 114,26 \%$, respectively $(p=0.161))$. Categorizing participants according to the CPITN, 20\% $(n=295)$ of participants had periodontal health (score 0$) ; 71 \%(n=1032)$ BOP or BOP and calculus (scores $1 \& 2)^{1} ; 8 \%(n=123)$ PD $4-5 \mathrm{~mm}$ (score 3$)$; and < $1 \%(\mathrm{n}=2) \mathrm{PD} \geq 6 \mathrm{~mm}$ (score 4) [21, 22].

\section{Oral pain status}

Very few participants $(7 \%, n=107)$ reported having any oral pain (Table 3 ). Of those with pain, it was typically mild $(n=90 / 107,84 \%)$, although $16 \%(n=17 / 107)$ reported moderate or severe pain. Participants characterized their pain experience as toothache $(n=50 / 107$, $47 \%)$, loose tooth $(n=18 / 107,17 \%)$, sore or bleeding gums $(n=30 / 107,28 \%)$, lesions in the mouth $(n=12 /$ $107,11 \%)$, and jaw pain $(n=25 / 107,23 \%)$ (multiple responses possible). Treatment sought for pain was typically increased teeth brushing or gargling with warm salt water. Of those that used medicine to treat pain, $80 \%(n=16 / 20)$ of participants could not identify what type of medicine (multiple responses possible). Only $18 \%(n=19 / 107)$ of participants with pain sought care from a health practitioner. One-quarter $(n=350,25 \%)$ of participants reported having seen blood at some point in their life when cleaning their teeth, and almost half of those women $(n=161 / 345,47 \%)$ saw blood within in the last week.

\section{Risk factors}

Age, height, and gravidity were associated with gingivitis at the $p<0.05$ level. Most notably, gingivitis was more common in women who were older (OR 1.04, 95\% CI: $1.02,1.06$ for each year of age) and women of short (< $150 \mathrm{~cm}$ ) stature (clinical health 338/870 (38.9\%), clinical gingivitis 274/582 (47.1\%), OR 1.40, 95\% CI: 1.13, 1.73). Gingivitis was less common in women who had no previous pregnancies (clinical health 260/870 (30.0\%), clinical gingivitis 143/582 (24.6\%), OR 0.78, 95\% CI: $0.61,0.99)$, although this association became insignificant when controlled for age (aOR 0.89, 95\% CI: 0.68, 1.15). Ethnic group (Pahadi or Madeshi), house roof construction material, and presence of a latrine were associated with gingivitis at the $p<0.1$ level. Several characteristics known from previous studies to be associated with gingivitis are not listed in Table 1 because the prevalence of these factors was reported at or near $0 \%$, for example, smoking and other tobacco use, alcohol use, and hypertension.

\section{Dental hygiene behaviors}

Nearly all participants reported teeth cleaning, most only once per day $(n=1030,73 \%)$ and in the morning $(n=$ 
Table 3 Oral pain status of participants

\begin{tabular}{|c|c|c|c|c|}
\hline Characteristic & All & Clinical health & Clinical gingivitis & OR $(95 \% C l)^{a}$ \\
\hline \multicolumn{5}{|l|}{ Current oral pain status $^{d}$} \\
\hline None & $1312(92.5)$ & $794(93.2)$ & $518(91.4)$ & Ref \\
\hline Mild & $90(6.3)$ & $47(5.5)$ & $43(7.6)$ & $1.40(0.91,2.15)$ \\
\hline Moderate or severe & $17(1.2)$ & $11(1.3)$ & $6(1.1)$ & $0.84(0.31,2.27)$ \\
\hline \multicolumn{5}{|l|}{ Type of pain ${ }^{b}$} \\
\hline Toothache & $50(46.7)$ & $29(50.0)$ & $21(42.9)$ & $0.75(0.35,1.61)$ \\
\hline Loose tooth & $18(16.8)$ & $7(12.1)$ & $11(22.4)$ & $2.11(0.75,5.95)$ \\
\hline Sore or bleeding gums & $30(28.0)$ & $10(17.2)$ & $20(40.8)$ & $3.31(1.36,8.05)$ \\
\hline Lesions in the mouth & $12(11.2)$ & $6(10.3)$ & $6(12.2)$ & $1.21(0.36,4.02)$ \\
\hline Jaw pain & $25(23.4)$ & $11(19.0)$ & $14(28.6)$ & $1.71(0.69,4.22)$ \\
\hline \multicolumn{5}{|l|}{ Treatment sought for pain ${ }^{b}$} \\
\hline Brushing & $48(44.9)$ & $30(51.7)$ & $18(36.7)$ & $0.54(0.25,1.18)$ \\
\hline Medicine & $20(18.7)$ & $14(24.1)$ & $6(12.2)$ & $0.44(0.15,1.25)$ \\
\hline Gargle with warm salt water & $40(37.4)$ & $23(39.7)$ & $17(34.7)$ & $0.81(0.37,1.78)$ \\
\hline Change eating or drinking habits & $16(15.0)$ & $6(10.3)$ & $10(20.4)$ & $2.22(0.74,6.64)$ \\
\hline Visited a health practitioner & $19(17.8)$ & $13(22.4)$ & $6(12.2)$ & $0.48(0.17,1.39)$ \\
\hline Other & $20(18.7)$ & $9(15.5)$ & $11(22.4)$ & $1.58(0.59,4.19)$ \\
\hline \multicolumn{5}{|l|}{ Saw blood when cleaning teeth (ever) ${ }^{c}$} \\
\hline No & $1069(75.3)$ & $713(83.7)$ & $356(62.8)$ & Ref \\
\hline Yes & $350(24.7)$ & $139(16.3)$ & $211(37.2)$ & $3.04(2.37,3.90)$ \\
\hline \multicolumn{5}{|c|}{ Saw blood when cleaning teeth (in last week) ${ }^{c}$} \\
\hline No & $183(53.2)$ & $86(63.2)$ & $97(46.6)$ & Ref \\
\hline Yes & $161(46.8)$ & $50(36.8)$ & $111(53.4)$ & $1.97(1.26,3.06)$ \\
\hline \multicolumn{5}{|l|}{ Clench or grind teeth while sleeping } \\
\hline No & $1352(95.2)$ & $813(95.4)$ & $539(94.9)$ & Ref \\
\hline Yes & $68(4.8)$ & $39(4.6)$ & $29(5.1)$ & $1.12(0.69,1.84)$ \\
\hline \multicolumn{5}{|l|}{ Wake up with tired jaws } \\
\hline No & $1327(93.5)$ & $798(93.7)$ & $529(93.1)$ & Ref \\
\hline Yes & $93(6.5)$ & $54(6.3)$ & $39(6.9)$ & $1.09(0.71,1.67)$ \\
\hline
\end{tabular}

Data presented at No. (\%) unless otherwise noted

annadjusted odds ratio and $95 \%$ confidence interval as appropriate

${ }^{\mathrm{b}}$ Multiple responses possible

'Participants seeing blood in the last week are a subset of those reporting having ever seen blood while cleaning teeth

dOral pain defined as none (no pain), mild (only a bother), and moderate or severe (prohibits daily activities)

Statistically significant for an odds ratio are in bold

1407, 99\%) (multiple responses possible) (Table 4). More than three quarters $(n=1113 / 1412,79 \%)$, however, indicated that, optimally, a person should clean their teeth twice or more per day. Average teeth cleaning time was $10($ median $=5)$ minutes. Three-quarters $(n=1044,74 \%)$ of participants used a toothbrush, and a large proportion reported using datiwan (दतविन) ( $n=626,43 \%)$, a local teeth cleaning instrument fashioned from twigs of a variety trees (multiple responses possible). We identified at least 18 species of tree - including bamboo, neem, Indian rosewood, mulberry, and guava - that are used for datiwan in this community. Datiwan use was much more common among families originating in the plains region of Nepal (Madeshi ethnic group), where almost half of women ( $n=618 / 1346,46 \%)$ reported using datiwan, than the hills region (Pahadi group), where only $8 \%$ ( $n=$ $8 / 106)$ reported use (OR 10.39, 95\% CI: 5.02, 21.55). More than half of participants ( $n=817,58 \%)$ used toothpaste, $16 \%(n=229)$ toothpowder, and $28 \%$ ( $n=$ 393) neither toothpaste nor toothpowder (multiple responses possible). Of women that used toothpaste, 55\% $(n=584)$ had a toothpaste brand that contained fluoride. Dental floss and oral rinse use were nearly non-existent $(<1 \%)$. Very few women reported changing any of their 
Table 4 Dental hygiene behaviors of participants

\begin{tabular}{|c|c|c|c|c|}
\hline Characteristic & All & Clinical health & Clinical gingivitis & Mean difference / OR $(95 \% \mathrm{Cl})^{\mathrm{a}}$ \\
\hline \multicolumn{5}{|c|}{ Cleaning frequency (times per day) } \\
\hline 1 & $1030(72.6)$ & $618(72.6)$ & $412(72.7)$ & Ref \\
\hline$\geq 2$ & $388(27.4)$ & $233(27.4)$ & $155(27.3)$ & $1.00(0.79,1.27)$ \\
\hline \multicolumn{5}{|c|}{ Cleaning frequency (times per week) } \\
\hline $1-6$ & $56(3.9)$ & $28(3.3)$ & $28(4.9)$ & Ref \\
\hline $7-13$ & $1148(80.9)$ & $702(82.4)$ & $446(78.7)$ & $0.64(0.37,1.09)$ \\
\hline$\geq 14$ & $215(15.2)$ & $122(14.3)$ & $93(16.4)$ & $0.76(0.42,1.37)$ \\
\hline \multicolumn{5}{|l|}{ Cleaning timing ${ }^{b}$} \\
\hline Morning & $1407(99.2)$ & 845 (99.4) & $562(98.9)$ & $0.55(0.17,1.82)$ \\
\hline Afternoon & $172(12.1)$ & $96(11.3)$ & $76(13.4)$ & $1.22(0.88,1.68)$ \\
\hline Evening & $291(20.5)$ & $175(20.5)$ & $116(20.4)$ & $0.99(0.76,1.29)$ \\
\hline After meals & 239 (16.8) & $140(16.4)$ & 99 (17.4) & $1.07(0.81,1.42)$ \\
\hline After sweets & $16(1.1)$ & $9(1.1)$ & $7(1.2)$ & $1.17(0.43,3.16)$ \\
\hline \multicolumn{5}{|l|}{ Cleaning duration } \\
\hline Minutes (mean $\pm \mathrm{SD}$ ) & $10.0 \pm 10.3$ & $10.1 \pm 10.3$ & $9.7 \pm 10.3$ & $-0.4(-1.5,0.7)$ \\
\hline \multicolumn{5}{|l|}{ Cleaning duration (mins) } \\
\hline $1-2$ & $90(6.4)$ & $50(5.9)$ & $40(7.1)$ & Ref \\
\hline $3-10$ & $1070(75.7)$ & $645(76.2)$ & $425(75.0)$ & $0.82(0.53,1.27)$ \\
\hline $11-29$ & $105(7.4)$ & $65(7.7)$ & $40(7.1)$ & $0.77(0.43,1.36)$ \\
\hline$\geq 30$ & $149(10.5)$ & $87(10.3)$ & $62(10.9)$ & $0.89(0.53,1.51)$ \\
\hline \multicolumn{5}{|l|}{ Instrument use ${ }^{b}$} \\
\hline Toothbrush & $1044(73.5)$ & $635(74.5)$ & $409(72.0)$ & $0.88(0.69,1.12)$ \\
\hline Datiwan & $626(43.1)$ & $359(41.3)$ & $267(45.9)$ & $1.21(0.98,1.49)$ \\
\hline Finger & $210(14.8)$ & $126(14.8)$ & $84(14.8)$ & $1.00(0.74,1.35)$ \\
\hline \multicolumn{5}{|c|}{ Brush replacement frequency (months) } \\
\hline $1-2$ & $720(69.8)$ & $442(71.1)$ & $278(68.0)$ & Ref \\
\hline $3-6$ & $275(26.7)$ & $156(25.1)$ & $119(29.1)$ & $1.21(0.91,1.61)$ \\
\hline$\geq 7$ & $36(3.5)$ & $24(3.9)$ & $12(2.9)$ & $0.79(0.39,1.62)$ \\
\hline \multicolumn{5}{|c|}{ Dentifrice use (at least once per week) } \\
\hline Toothpaste & $817(57.5)$ & $497(58.3)$ & $320(56.3)$ & $0.92(0.74,1.14)$ \\
\hline Danta munjhan powder & $229(16.1)$ & $138(16.2)$ & $91(16.0)$ & $0.99(0.74,1.32)$ \\
\hline Charcoal & $39(2.7)$ & $23(2.7)$ & $16(2.8)$ & $1.04(0.55,2.00)$ \\
\hline Other & $54(3.8)$ & $30(3.5)$ & $24(4.2)$ & $1.21(0.70,2.09)$ \\
\hline \multicolumn{5}{|l|}{ Fluoride in toothpaste } \\
\hline No & $455(42.8)$ & $274(42.2)$ & $181(43.6)$ & Ref \\
\hline Yes & $584(54.9)$ & $361(55.6)$ & $223(53.7)$ & $0.94(0.73,1.20)$ \\
\hline Don't know & $25(2.3)$ & $14(2.2)$ & $11(2.7)$ & $1.19(0.53,2.68)$ \\
\hline \multicolumn{5}{|c|}{ New behaviors adopted during pregnancy ${ }^{\mathrm{b}}$} \\
\hline More teeth cleaning & $54(3.8)$ & $35(4.1)$ & $19(3.3)$ & $0.81(0.46,1.43)$ \\
\hline Less teeth cleaning & $5(0.4)$ & $4(0.5)$ & $1(0.2)$ & $0.37(0.04,3.35)$ \\
\hline New instrument & $28(2.0)$ & $13(1.5)$ & $15(2.6)$ & $1.75(0.83,3.71)$ \\
\hline New dentifrice & $8(0.6)$ & $6(0.7)$ & $2(0.4)$ & $0.50(0.10,2.48)$ \\
\hline
\end{tabular}

Data presented as No. (\%) unless otherwise noted

${ }^{\mathrm{a}}$ T-test or unadjusted odds ratio and $95 \%$ confidence interval as appropriate

${ }^{\mathrm{b}}$ Multiple responses possible 
dental hygiene behaviors at the start of their current pregnancy. In general, these dental hygiene characteristics were not strongly associated with gingivitis; although, at the $p<0.1$ significance level, the condition was more common in those who used datiwan (clinical health 359/870 (41.3\%), clinical gingivitis 267/582 (45.9\%), OR 1.21, 95\% CI: 0.98, 1.49).

\section{Dental health care seeking behaviors}

While the overwhelming majority of participants $(n=$ $1248,88 \%)$ had never visited a dentist, those that had sought professional care $(n=171,12 \%)$ most often indicated that doing so was prompted by toothache $(n=94 /$ $171,55 \%)$ or dental caries $(n=106 / 171,62 \%)$ (multiple responses possible) (Additional file 1: Table S1). Barriers to visiting a dentist included not knowing about dentists ( $n=257,18 \%)$ or where to seek care $(n=224,16 \%)$, but a large proportion also indicated there was no need for them to visit a dentist $(n=1160,82 \%)$ (multiple responses possible). Commonly reported sources of dental health information were family $(n=1103,78 \%)$, radio $(n=760,54 \%)$, school $(n=650,46 \%)$, friends $(n=457$, $32 \%)$, and television $(n=216,15 \%)$. Likelihood of gingivitis was lower among women who reported receiving dental health information from radio (clinical health 474/852 (55.6\%), clinical gingivitis 286/568 (50.4\%), OR $0.81,95 \%$ CI: $0.65,1.00$ ) or television (clinical health $145 / 852$ (17.0\%), clinical gingivitis 71/568 (12.5\%), OR $0.70,95 \% \mathrm{CI}: 0.51,0.95)$ and among women who said they had no need to visit the dentist (clinical health 709/ 852 (83.2\%), clinical gingivitis $451 / 568$ (79.4\%), OR 0.78 , 95\% CI: 0.59, 1.02). Risk of gingivitis was significantly higher among women reporting that cost of dental was too expensive (clinical health $17 / 851$ (2.0\%), clinical gingivitis 24/568 (4.2\%), OR 2.16, 95\% CI: 1.15, 4.07).

\section{Dental health attitudes}

Participants reported many different reasons why they cleaned their teeth (Additional file 1: Table S2). Most frequently mentioned barriers to more frequent cleaning included never being taught to clean their teeth $(n=$ $518,37 \%)$, not thinking additional teeth cleaning was necessary $(n=531,37 \%)$, too much bother $(n=448$, $32 \%)$, teeth are not dirty $(n=251,18 \%)$, not enough time ( $n=257,18 \%$ ), and no one else is doing so around me ( $n=216,15 \%)$ (multiple responses possible). Gingivitis was less common among women who said that they clean their teeth because they were taught to do so (clinical health 359/852 (42.1\%), clinical gingivitis 210/568 (37.0\%), OR 0.81, 95\% CI: 0.65, 1.00). Gingivitis was also less common among those that reported that reasons preventing them from brushing their teeth included: cleaning their teeth does not help (clinical health 78/852 (9.2\%), clinical gingivitis 36/567 (6.4\%), OR 0.67, 95\% CI:
$0.45,1.01$ ) or is not necessary (clinical health $334 / 851$ (39.3\%), clinical gingivitis 197/568 (34.7\%), OR 0.82, 95\% CI: 0.66, 1.03). Gingivitis was more common among those that reported that reasons preventing them from brushing their teeth included: no one else around me cleans their teeth (clinical health 109/852 (12.8\%), clinical gingivitis 107/567 (18.9\%), OR 1.59, 95\% CI: $1.19,2.12)$ or cleaning teeth is too much of a bother (clinical health 252/852 (29.6\%), clinical gingivitis 195/ 567 (34.4\%), OR 1.25, 95\% CI: 0.99, 1.57).

\section{Multivariable analysis}

In the final regression model, the odds of gingivitis increased significantly by $3 \%$ (aOR $1.03,95 \%$ CI: 1.00 , 1.06) for each year of age (Table 5). Odds of gingivitis were lower among the Madeshi vs. Pahadi groups (aOR 0.57, 95\% CI: $0.37,0.88$ ). Odds of gingivitis were $43 \%$ higher for women of short stature $(<150 \mathrm{~cm})(\mathrm{aOR} 1.43$, 95\% CI: 1.14, 1.79). Participant self-report that cost inhibits how often they visit the dentist was associated with increased risk of gingivitis (aOR 2.13, 95\% CI: 1.09, 4.15). As reasons for why participants did not clean their teeth more often, higher odds of gingivitis were seen among those reporting no one else does (aOR 1.69, 95\% CI: 1.24, 2.31) and lower odds among those reporting that teeth cleaning doesn't help (aOR 0.53, 95\% CI: $0.34,0.83$ ), and don't think it's necessary (aOR 0.78, 95\% CI: 0.62, 0.99).

\section{Discussion}

We found approximately $40 \%$ of women in this population to have gingivitis and $79 \%$ to have $\geq 1$ site with BOP. Although bleeding on probing was highly prevalent, very few women had signs of periodontitis. Periodontal probing depths of $>3 \mathrm{~mm}$ were observed in $9 \%$ of women, consistent with documented increases in gingivitis and probing depths during pregnancy [23]. Participants had minimal recession, and CAL estimates, in both women with and without disease, were driven primarily by probing depth. This may be a result of the young age distribution of the population. CPITN scores from our study fit with the disease burden seen in the most recent national survey of periodontal disease in Nepal [10]. In other populations, higher levels of gingival inflammation and probing depths were observed among pregnant women during pregnancy [23-25].

Age was positively associated with periodontal disease, although many common risk factors for periodontal disease, such as smoking, alcohol use, and hypertension and other chronic diseases were low or nonexistent in this population. The ethnic composition of the study population was relatively homogenous, divided across the Pahadi group (people originally from the hills) and Madeshi group (people originally from the plains)) 
Table 5 Association between participant characteristics and clinical gingivitis status

\begin{tabular}{|c|c|}
\hline & $\begin{array}{l}\text { Clinical gingivitis } \\
\text { OR }(95 \% \mathrm{Cl})\end{array}$ \\
\hline \multicolumn{2}{|l|}{ Model 1 - Maternal characteristics $(n=1452)$} \\
\hline Age & $1.03(1.01,1.06)$ \\
\hline Maternal stature $(<150 \mathrm{~cm})$ & $1.42(1.15,1.75)$ \\
\hline Primiparous & $0.89(0.69,1.17)$ \\
\hline \multicolumn{2}{|c|}{ Model 2 - Model 1 + Oral hygiene behaviors $(n=1452)$} \\
\hline Age & $1.03(1.01,1.06)$ \\
\hline Maternal stature $(<150 \mathrm{~cm})$ & $1.39(1.12,1.73)$ \\
\hline Primiparous & $0.90(0.69,1.18)$ \\
\hline Teeth cleaning frequency & $0.71(0.46,1.10)$ \\
\hline Use of datiwan to clean teeth & $1.15(0.92,1.42)$ \\
\hline \multicolumn{2}{|c|}{ Model 3 - Models $1-2+$ Oral health knowledge \& attitudes $(n=1417)$} \\
\hline Age & $1.03(1.01,1.06)$ \\
\hline Maternal stature $(<150 \mathrm{~cm})$ & $1.43(1.14,1.78)$ \\
\hline Primiparous & $0.93(0.71,1.23)$ \\
\hline Teeth cleaning frequency & $0.66(0.38,1.15)$ \\
\hline Use of datiwan to clean teeth & $1.10(0.88,1.38)$ \\
\hline No need to visit dentist & $0.82(0.62,1.09)$ \\
\hline Too much money to visit dentist & $2.20(1.13,4.28)$ \\
\hline Radio a source of dental health information & $0.93(0.74,1.18)$ \\
\hline TV a source of dental health information & $0.76(0.55,1.05)$ \\
\hline Clean teeth because taught to do so & $0.81(0.65,1.02)$ \\
\hline Prevent cleaning - it's a bother & $1.28(1.01,1.62)$ \\
\hline Prevent cleaning - no one else does & $1.70(1.25,2.32)$ \\
\hline Prevent teeth cleaning - doesn't help & $0.53(0.34,0.82)$ \\
\hline $\begin{array}{l}\text { Prevent teeth cleaning - don't think it's } \\
\text { necessary }\end{array}$ & $0.79(0.62,1.00)$ \\
\hline \multicolumn{2}{|c|}{ Final model - Models 1-3+ Socioeconomic status $(n=1417)$} \\
\hline Age & $1.03(1.00,1.06)$ \\
\hline Maternal stature $(<150 \mathrm{~cm})$ & $1.43(1.14,1.79)$ \\
\hline Primiparous & $0.91(0.69,1.20)$ \\
\hline Teeth cleaning frequency & $0.70(0.40,1.21)$ \\
\hline Use of datiwan to clean teeth & $1.13(0.90,1.43)$ \\
\hline No need to visit dentist & $0.82(0.61,1.09)$ \\
\hline Too much money to visit dentist & $2.13(1.09,4.15)$ \\
\hline Radio a source of dental health information & $0.93(0.74,1.18)$ \\
\hline TV a source of dental health information & $0.74(0.53,1.03)$ \\
\hline Clean teeth because taught to do so & $0.83(0.66,1.05)$ \\
\hline Prevent cleaning - it's a bother & $1.25(0.99,1.58)$ \\
\hline Prevent cleaning - no one else does & $1.69(1.24,2.31)$ \\
\hline Prevent teeth cleaning - doesn't help & $0.53(0.34,0.83)$ \\
\hline
\end{tabular}

Table 5 Association between participant characteristics and clinical gingivitis status (Continued)

\begin{tabular}{|c|c|}
\hline & $\begin{array}{l}\text { Clinical gingivitis } \\
\text { OR }(95 \% \mathrm{Cl})\end{array}$ \\
\hline $\begin{array}{l}\text { Prevent teeth cleaning - don't think } \\
\text { it's necessary }\end{array}$ & $0.78(0.62,0.99)$ \\
\hline Madeshi cultural group & $0.57(0.37,0.88)$ \\
\hline Sturdy roof construction & $0.85(0.56,1.31)$ \\
\hline Latrine in home & $0.91(0.72,1.15)$ \\
\hline
\end{tabular}

Statistically significant for an odds ratio are in bold

indigenous to Nepal. Risk factors for poor oral health that were prevalent in our population included rural location, low-socioeconomic status, and poor oral hygiene, specifically that women typically only cleaned their teeth once per day (sometimes with datiwan, rather than a toothbrush), few used fluorinated toothpaste, almost none flossed or used oral rinse, and access to professional dental health care was rare [26-28].

Some oral hygiene habits identified in this study were described similarly elsewhere, such as the practice of cleaning teeth only once per day (typically in the morning), but other habits were different, such as the high prevalence of datiwan use in our population, likely driven by behaviors common to the Madeshi ethnic group and the Terai region [29-31]. Datiwan use was the main driver of the high teeth cleaning time, as it is common practice in this population to use datiwan over an extended period during the morning routine. A large majority of women in this community had never visited a dentist or received any dental care [29]. A higher proportion of participants from this study reported having pain due to bleeding or swollen gums (relative to tooth pain) compared to those in an urban population in Eastern Nepal [30]. These findings are complimented by a qualitative study conducted in this population by our research team, which found that women were receptive to switching their routine to brushing twice daily with toothpaste when instructed by a health worker [32].

Although a wide range of potential risk factors for gingivitis were examined, other important factors, including some known to be causative, were not represented, including systemic conditions, such as diabetes mellitus or psychosocial factors, such as stress [33]. Although chronic diseases are likely under-diagnosed in this rural population, we suspect the prevalence of chronic disease to be low, given previous studies of risk factors in this population, and the young age of women in this study [34]. Undernutrition was not directly considered in the analysis, but was likely prevalent among women in the study population [35].

This study had several limitations. Attachment loss, a common measure of periodontitis, was not included in 
our outcome definition because it was not collected for all sites. Other indicators of periodontal disease, such as gingival and plaque scores, were not measured due to visit time constraints and variability in exam conditions, particularly in the amount of lighting. Although the validity of PD measurements for each community-based oral health worker was assessed relative to a dentist, reliability between or within the oral health workers was not evaluated.

\section{Conclusion}

This study found $40 \%$ of women of childbearing age to have signs of gingivitis. Oral hygiene behaviors were less frequent than and varied from recommended practices and access to dental health services was uncommon. Age and short maternal stature were among the risk factors for gingivitis. These findings were consistent with the burden of periodontal disease measured by other studies in Nepal, although some important differences were identified in this population, including the prevalent use of datiwan for teeth cleaning [12, 31]. Data from this study will allow for evaluation of the role of periodontal disease in adverse pregnancy outcomes, such as preterm birth and low birth weight. Future studies could describe in further detail the severity and prevalence of periodontal disease, for example, by utilizing gingival and plaque indices, or evaluate the effectiveness of community-based interventions to prevent oral disease in women and mothers in Nepal.

\section{Endnotes}

${ }^{1}$ Presence of calculus was not assessed by this study.

\section{Additional file}

Additional file 1: Table S1. Dental health care seeking behaviors of participants. Table S2. Dental health attitude of participants. (DOCX 26 kb)

\section{Abbreviations}

aOR: Adjusted odds ratios; BMl: Body mass index; BOP: Bleeding on probing; CAL: Clinical attachment loss; CEJ: Cemento-enamel junction; Cl: Confidence intervals; CPITN: Community Periodontal Index of Treatment Needs: GM: Gingival margin; NOHCS: Nepal Oral Health Cohort Study; NOMS: Nepal Oil Massage Study; OR: Odds ratio; PD: Probing depth; SD: Standard deviation

\section{Acknowledgements}

Not applicable.

\section{Funding}

This study was supported by the National Institute for Child Health and Development (HD060712) and the Bill \& Melinda Gates Foundation (OPP1084399, OPP1131701).

\section{Availability of data and materials}

All data files, codebooks, and related manuscripts are available from the JHU Data Archive (https://doi.org/10.7281/T1/ZPGBJW).

\section{Authors' contribution}

DJE conceptualized and designed the study jointly with other authors, developed field implementation protocols, oversaw implementation in the field, conducted the analysis and wrote the manuscript. BR contributed to field implementation, quality control of field procedures, and provided comments on the manuscript. SKK helped conceptualize and design the study jointly with other authors, oversaw field implementation and ensured quality, provided comments on the manuscript. SCL contributed to the study design and overall implementation in the field, helped with interpretation of the results, and provided comments on the manuscript. NKA helped design the study, provided input on the data collection approach and content, training of oral health workers, and provided comments on the manuscript. MAR conceptualized and designed the study jointly with other authors, gave comments on the manuscript. JK contributed to the study design, quality of data collected, and gave comments on the manuscript. LCM conceptualized and designed the study jointly with other authors, obtained funding the study, oversaw implementation approach, obtained ethical approvals, advised on analytic approach, and edited the manuscript. All authors read and approved the final manuscript.

\section{Ethics approval and consent to participate}

Ethical approval was obtained from the Institutional Review Board (IRB) at the Johns Hopkins Bloomberg School of Public Health (\#6297) and from the independent Ethical Review Board of the Nepal Health Research Council (NHRC) (\#188/2015). All enrolled persons in this study were married. All provided written consent for participation, including married women under 18 years of age, who were considered emancipated minors according to local and international ethical review boards overseeing this project. NHRC approved the age of consent for participation in this study as at least 15 years.

\section{Consent for publication}

Not applicable.

\section{Competing interests}

The authors declare that they have no competing interests.

\section{Publisher's Note}

Springer Nature remains neutral with regard to jurisdictional claims in published maps and institutional affiliations.

\section{Author details}

'Department of International Health, Johns Hopkins Bloomberg School of Public Health, Baltimore, MD, USA. ${ }^{2}$ Nepal Nutrition Intervention Project, Sarlahi (NNIPS), Krishna Galli, Lalitpur, Kathmandu, Nepal. ${ }^{3}$ Department of Dentistry, Institute of Medicine, Tribhuhvan University, Kathmandu, Nepal. ${ }^{4}$ Department of Advanced Oral Sciences and Therapeutics, University of Maryland School of Dentistry, Baltimore, MD, USA.

Received: 10 April 2018 Accepted: 27 November 2018

Published online: 05 January 2019

\section{References}

1. Brown LJ, Loe H. Prevalence, extent, severity and progression of periodontal disease. Periodontology 2000. 1993;2:57-71

2. Papapanou PN. Periodontal diseases: epidemiology. Ann Periodontol. 1996; 11:1-36.

3. Tanner ACR, Kent R, Van Dyke T, Sonis ST, Murray LA. Clinical and other risk indicators for early periodontitis in adults. J Periodontol. 2005;764:573-81.

4. Kwan S, Petersen PE. Oral health: equity and social determinants. In: SKA BE, editor. Equity, social determinants and public health programmes. Geneva: World Health Organization; 2010. p. 159-76.

5. Offenbacher S, Katz V, Fertik G, Collins J, Boyd D, Maynor G, et al. Periodontal infection as a possible risk factor for preterm low birth weight. $J$ Periodontol. 1996:6710(Suppl):1103-13.

6. Jin LJ, Armitage GC, Klinge B, Lang NP, Tonetti M, Williams RC. Global oral health inequalities: task group--periodontal disease. Adv Dent Res. 2011;232: 221-6. 
7. Kandelman D, Arpin S, Baez RJ, Baehni PC, Petersen PE. Oral health care systems in developing and developed countries. Periodontology 2000. 2012; 601:98-109.

8. Corbet EF, Leung WK. Epidemiology of periodontitis in the Asia and Oceania regions. Periodontology 2000. 2011;561:25-64.

9. van Palenstein Helderman W, Groeneveld A, Jan Truin G, Kumar Shrestha B, Bajracharya M, Stringer R. Analysis of epidemiological data on oral diseases in Nepal and the need for a national oral health survey. Int Dent J. 1998; 481:56-61.

10. Yee R, Mishra P. Nepal National Oral Health 'pathfinder' survey 2004; 2004.

11. Yee R, David J, Lama D. The periodontal health of Nepalese schoolchildren. Community Dent Health. 2009;264:250-6.

12. David J, Yee R, Lama D. The periodontal health of adult Nepalese. Oral Health Prev Dent. 2011;91:67-81.

13. Ide M, Papapanou PN. Epidemiology of association between maternal periodontal disease and adverse pregnancy outcomes--systematic review. J Periodontol. 2013;844(Suppl):S181-94.

14. Armitage GC. Development of a classification system for periodontal diseases and conditions. Ann Periodontol. 1999:41:1-6.

15. Chapple ILC, Mealey BL, Van Dyke TE, Bartold PM, Dommisch H, Eickholz P, et al. Periodontal health and gingival diseases and conditions on an intact and a reduced periodontium: consensus report of workgroup 1 of the 2017 world workshop on the classification of periodontal and Peri-implant diseases and conditions. J Periodontol. 2018;89(Suppl 1):S74-s84.

16. Trombelli L, Farina R, Silva CO, Tatakis DN. Plaque-induced gingivitis: case definition and diagnostic considerations. J Periodontol. 2018;89(Suppl 1):S46-s73.

17. Holtfreter B, Albandar JM, Dietrich T, Dye BA, Eaton KA, Eke PI, et al. Standards for reporting chronic periodontitis prevalence and severity in epidemiologic studies: proposed standards from the joint EU/USA periodontal epidemiology working group. J Clin Periodontol. 2015:425:407-12

18. Papapanou PN, Sanz M, Buduneli N, Dietrich T, Feres M, Fine DH, et al. Periodontitis: consensus report of workgroup 2 of the 2017 world workshop on the classification of periodontal and Peri-implant diseases and conditions. J Periodontol. 2018;89(Suppl 1):S173-s82.

19. Tonetti MS, Greenwell H, Kornman KS. Staging and grading of periodontitis: framework and proposal of a new classification and case definition. J Periodontol. 2018;89(Suppl 1):S159-s72.

20. World Health Organization. Epidemiology, etiology, and prevention of periodontal diseases. World Health Organization technical report series. 1978.

21. World Health Organization. Oral health surveys basic methods. 5th ed. Geneva, Switzerland: World Health Organization; 2013.

22. World Health Organization. Oral Health Country/Area Profile Programme. WHO Collaborating Centres, Malmo University, Sweden and Niigata University, Japan; 2017.

23. Gonzalez-Jaranay M, Tellez L, Roa-Lopez A, Gomez-Moreno G, Moreu G. Periodontal status during pregnancy and postpartum. PLoS One. 2017;125: e0178234.

24. Laine MA. Effect of pregnancy on periodontal and dental health. Acta Odontol Scand. 2002;605:257-64.

25. Figuero E, Carrillo-de-Albornoz A, Martin C, Tobias A, Herrera D. Effect of pregnancy on gingival inflammation in systemically healthy women: a systematic review. J Clin Periodontol. 2013;405:457-73.

26. Steinberg BJ, Hilton IV, lida H, Samelson R. Oral health and dental care during pregnancy. Dent Clin N Am. 2013;572:195-210.

27. Wagle M, Trovik TA, Basnet P, Acharya G. Do dentists have better oral health compared to general population: a study on oral health status and oral health behavior in Kathmandu, Nepal. BMC Oral Health. 2014;14:23.

28. Knevel RJ. Training rural women to improve access to oral health awareness programmes in remote villages in Nepal. Int J Dent Hyg. 2010;84:286-93.

29. Thapa P, Aryal KK, Mehata S, Vaidya A, Jha BK, Dhimal M, et al. Oral hygiene practices and their socio-demographic correlates among Nepalese adult: evidence from non communicable diseases risk factors STEPS survey Nepal 2013. BMC oral health. 2016;161:105.

30. Dali M, Laleet R. A study on oral health knowledge, attitude, and practice among population of Siswani Jahada VDC in Biratnagar City, Nepal - a questionnaire survey. Asian Pac J Health Sci. 2014;12:51-6.

31. Buunk-Werkhoven YA, Dijkstra A, Bink P, van Zanten S, van der Schans CP. Determinants and promotion of oral hygiene behaviour in the Caribbean and Nepal. Int Dent J. 2011;615:267-73.

32. Lubon AJ, Erchick DJ, Khatry SK, LeClerq SC, Agrawal NK, Reynolds MA, et al. Oral health knowledge, behavior, and care seeking among pregnant and recently-delivered women in rural Nepal: a qualitative study. BMC oral health. 2018;181:97.

33. Petersen PE, Ogawa $H$. The global burden of periodontal disease: towards integration with chronic disease prevention and control. Periodontology 2000. 2012;601:15-39.

34. Khan RJ, Stewart CP, Christian P, Schulze KJ, Wu L, Leclerq SC, et al. A crosssectional study of the prevalence and risk factors for hypertension in rural Nepali women. BMC Public Health. 2013;13:55.

35. Campbell RK, Talegawkar SA, Christian P, LeClerq SC, Khatry SK, Wu LS, et al. Seasonal dietary intakes and socioeconomic status among women in the Terai of Nepal. J Health Popul Nutr. 2014;322:198-216.
Ready to submit your research? Choose BMC and benefit from:

- fast, convenient online submission

- thorough peer review by experienced researchers in your field

- rapid publication on acceptance

- support for research data, including large and complex data types

- gold Open Access which fosters wider collaboration and increased citations

- maximum visibility for your research: over $100 \mathrm{M}$ website views per year

At $\mathrm{BMC}$, research is always in progress.

Learn more biomedcentral.com/submissions 